\title{
BMJ Open Public health programmes to promote mental health in young people: a systematic integrative review protocol
}

\author{
Nuwan Darshana Wickramasinghe (D) ,,2 Nelum Samarutilake, ${ }^{2,3}$ \\ Mihiri Chami Wettasinghe, ${ }^{4}$ Julie Feiler, ${ }^{5}$ Antony Morgan, ${ }^{5}$ Antonis A Kousoulis, ${ }^{6}$ \\ Tine Van Bortel (1D) 2,7
}

To cite: Wickramasinghe ND, Samarutilake N,

Wettasinghe MC, et al. Public health programmes to promote mental health in young people: a systematic integrative review protocol. BMJ Open 2020;10:e037241. doi:10.1136/ bmjopen-2020-037241

- Prepublication history and additional material for this paper are available online. To view these files, please visit the journal online (http://dx.doi. org/10.1136/bmjopen-2020037241).

Received 24 January 2020 Revised 30 July 2020 Accepted 21 August 2020

A) Check for updates

(C) Author(s) (or their employer(s)) 2020. Re-use permitted under CC BY-NC. No commercial re-use. See rights and permissions. Published by BMJ.

For numbered affiliations see end of article.

Correspondence to Dr Nuwan Darshana Wickramasinghe; nuwick74@yahoo.com

\section{ABSTRACT}

Introduction In light of the ever-growing mental health disease burden among young people worldwide, we aim to systematically review the global literature to identify the public health programmes targeted at promoting mental health and well-being in young people, the reported/ anticipated mental health-related outcomes of the implemented public health programmes and the reported facilitators and barriers in relation to the implementation of those public health programmes.

Methods and analysis A comprehensive literature search will be carried out in the following electronic bibliographic databases: MEDLINE, EMBASE, PsycINF0, Scopus, ASSIA, Web of Science, Global Health, AMED, Health Source and The Cochrane Library. Further, a manual search of the reference lists of eligible studies and reviews will be carried out. The search strategy will include combinations of three key blocks of terms, namely: 'young people', 'mental health' and 'public health programme', using database-specific subject headings and text words. Two reviewers will independently screen, assess data quality and extract data for synthesis. Disagreements at any stage will be resolved by consensus with the involvement of a third reviewer. Given the anticipated methodological pluralism of the potential eligible studies, we will provide a narrative synthesis of the findings on public health programmes aimed at promoting the mental health and well-being of young people according to identified thematic areas. Furthermore, a narrative synthesis of the reported facilitators and barriers in relation to the implementation of public health programmes will be provided.

Ethics and dissemination Given that the review findings will be focused on understanding the breadth and depth of the global research into public health programmes to promote mental health in young people with a particular emphasis on the facilitators and barriers of programmatic implementation, the findings will be of great value to inform future interventions, programmes and approaches to promote mental health and well-being of young people worldwide.

PROSPERO registration number CRD42018099551.

\section{INTRODUCTION}

Addressing young people's mental health needs is recognised as an urgent global public

\section{Strengths and limitations of this study}

- This proposed systematic integrative review will use a very sensitive search strategy focusing on understanding the breadth and depth of global research regarding public health programmes to promote mental health in young people.

- A significant number of relevant electronic databases will be searched using a wide range of search terms to comprehensively capture representative global literature.

- There will be no time restrictions on the literature search; however, searches will be limited to the English language and grey literature will not be included.

- This review will include a wide variety of study designs including peer-reviewed primary empirical quantitative (both experimental and observational), qualitative and mixed-methods studies.

- Owing to the anticipated high degree of methodological pluralism, an integrative literature review will be carried out to incorporate the research evidence generated using quantitative, qualitative and mixed methods.

health challenge. ${ }^{1}$ Systematic analysis of the global burden of disease in the 10-24years age group reveals that neuropsychiatric disorders accounted for $45 \%$ of years lost because of disability. ${ }^{2}$ Furthermore, global estimates suggest that $10 \%-20 \%$ of children and adolescents are reported to have mental health problems at any given time ${ }^{3}$ and, according to a recent meta-analysis, the pooled estimate of the prevalence of mental health problems in children and adolescents worldwide is $13.4 \%$. $^{4}$ Studies on age of onset distributions of mental health problems reveal that by age 14 , half of the mental health problems are established and by age 24, three-quarters of the mental health problems are established. ${ }^{5}$

Mental health problems can have devastating repercussions on different aspects of young people's health, leading to social 
isolation, stigmatisation and inability to effectively contribute to their individual as well as societal development and potential. Moreover, the resultant inequity of access to healthcare and education facilities are violations of their fundamental human rights. ${ }^{6-9}$ Against this backdrop, it is recommended to address mental health problems early in life, ${ }^{10}$ which ultimately results in better social and behavioural adjustment and academic achievements and also reduces emotional and behavioural problems including antisocial and criminal behaviours. ${ }^{911}$

In spite of the wealth of global evidence highlighting the link between mental health problems and healthrelated disability and their life-long negative effects in young people, $60 \%-70 \%$ of children and adolescents with mental health problems have not received early age appropriate interventions. ${ }^{9} 12$ Furthermore, the mental health needs of children and adolescents are often overlooked, especially in low-income and middleincome countries. ${ }^{13-15}$ Even though the global evidence suggests that young people worldwide have the highest disease burden in terms of mental ill health prevalence and incidence across the lifecycle with a disproportionate share of mental health-related disease burden, the accessibility of mental health services for young people is the poorest of all age groups due to a variety of reasons such as high rates of perceived stigma and embarrassment, low rates of mental health literacy and a preference for self-reliance. ${ }^{16-19}$

On the global health agenda, evidence substantiates that it is advantageous and efficient to combine both preventive and promotional programmes in mental health in the context of holistic public health programmes. Such combined programmes are especially beneficial in resource-poor settings in order to streamline the budgetary allocations for activities targeted at disease prevention and health promotion. ${ }^{20}$ The overall aim of those public health interventions is to promote or protect health or prevent ill health in order to deliver a net benefit to the wider public and/or specific population groups. Furthermore, public health approaches targeted at preventing health problems extending better care and safety to entire populations rather than merely focusing on individuals. ${ }^{20-22}$ Hence, it is vital to have a public health approach to address mental health problems going beyond the aspect of providing adequate treatment, which involves initiatives to support the promotive factors for mental well-being while suppressing the risk factors for mental health problems. ${ }^{23} 24$

Global evidence pertaining to young people's mental health promotion highlight the importance of adapting wider public health strategies focused on prevention of mental ill health and promotion of mental well-being in this age group. ${ }^{1} 13152526$ Hence, against the backdrop of the increasing global burden of mental health issues among young people, it is imperative to explore the different public health programmes conducted to promote young people's mental health and well-being in different cultural and geopolitical settings.
Even though there are published systematic reviews on different aspects related to mental health of young people, such as on identifying facilitators and barriers for mental health services, ${ }^{16}{ }^{18} 19$ school-based prevention programmes for mental health problems ${ }^{27}$ and reviews targeted on selected populations, such as young people in low-income and middle-income countries ${ }^{28}$ or adolescents, ${ }^{29}$ thus far, the relevant evidence base lacks comprehensive global literature reviews compiling evidence generated based on public health interventions to promote mental health in young people with a view to understanding the breadth and depth of relevant research. In the light of this important research vacuum against the backdrop of ever-increasing mental health problems burden among young people, it is an opportune time to review the global evidence on public mental health programmes for young people with an emphasis to identify the existing gaps in service provision and to highlight the examples of good practice from across the globe.

\section{Objective}

We aim to carry out a comprehensive global literature review in order to develop a consolidated overview of the evidence on public health programmes for mental health promotion in young people.

\section{Review questions}

The review will be conducted to answer the following review questions:

1. What are the public health programmes targeted at promoting mental health and well-being in young people?

2. What are the reported/anticipated mental healthrelated outcomes of the public health programmes implemented in young people?

3. What are the reported facilitators and barriers in relation to the implementation of public health programmes for mental health and well-being promotion in young people?

\section{METHODS AND ANALYSIS}

The Preferred Reporting Items for Systematic Reviews and Meta-Analyses (PRISMA) Protocols statement ${ }^{30}$ has been used in the preparation of this protocol (online supplemental file 1 ).

Provided that the objective of this review is to synthesise representative global literature on public health programmes for mental health promotion in young people and since the field of public health is characterised by a high degree of methodological pluralism, ${ }^{31}$ an integrative literature review will be carried out to incorporate the research evidence generated using quantitative, qualitative and mixed methods. ${ }^{32-34}$ This review protocol was initially developed in 2018 and it is intended to be completed in 2020. 


\section{Eligibility criteria}

This integrative review is aimed at identifying the peerreviewed publications on public health programmes for mental health promotion in young people according to the following eligibility criteria.

\section{Population}

Any study primarily focused on young people will be included in the review. WHO defines 'adolescents' as individuals in the 10-19 years of age group and 'youth' as the 15-24year age group. Together, adolescents and youth are referred to as young people, encompassing the ages of 10-24 years. ${ }^{35}$ Accordingly, the age group for young people will be considered as 10-24 years in this review. Studies focused on a mixed age group will be included in the review, if the programme objectives specifically mention young people as a target group.

\section{Intervention}

In this review, any public health programme implemented with an explicit general/specific objective of promoting or protecting mental health; and/or preventing or reducing adverse mental health outcome/s in young people will be included. Public health programmes targeted at mixed age groups (either universal or targeted) will also be included if the explicit programmatic objectives meet the above criterion. The programmes having young people as the level of intervention (rather than merely the target of intervention) will be included in the review. In multistage/multicomponent programmes having both population approach and individual approach, the stage/ component with population approach will be included in the review.

Studies focusing on public health interventions, which do not have any component of implemented programmes (eg, policies of governments and non-government organisations, laws and regulations) will not be included in the review.

Examples of potential inclusions are: health promotion/education programmes, coaching and other mental health interventions and services, surveillance, social marketing and school/college/university-based health programmes.

\section{Comparison}

Studies with or without any comparative group will be considered for the review.

\section{Primary outcome}

The primary outcome will be the reported/anticipated change in mental health and well-being (general or disease-specific) of young people. The outcome of interest could be expressed quantitatively (eg, measures of frequency such as prevalence or incidence, mean scores) or qualitatively.

\section{Secondary outcomes}

The secondary outcomes of the review will be the reported facilitators and barriers in relation to the implementation of public health programmes for mental health and wellbeing promotion in young people.

\section{Study design}

This integrative review will include peer-reviewed primary empirical quantitative (both experimental and observational), qualitative and mixed-methods studies.

\section{Context}

Any study reporting a public health programme conducted among young people as the level of intervention across the globe will be considered for the review (irrespective of whether community-based or institution/ any specific setting based).

\section{Search strategy}

\section{Data sources}

We will carry out a comprehensive literature search and the electronic search will include bibliographic database search using MEDLINE, EMBASE, PsycINFO, Scopus, ASSIA, Web of Science, Global Health, AMED, Health Source and The Cochrane Library (Cochrane Database of Systematic Reviews, Cochrane Central Register of Controlled Trials, Cochrane Methodology Register). There will be no time restrictions applied to any electronic database search (ie, the search will be conducted from the first available date to the date of search for each database). The electronic search will be supplemented by manual search of the reference lists of eligible studies and reviews.

\section{Search terms}

The search strategy will include combinations of three key blocks of terms, namely: 'young people', 'mental health' and 'public health programme' (online supplemental file 2), using a combination of medical subject headings terms and text words.

The search strategy will be tailored appropriately for each database. There will be no time restrictions, however, searches will be limited to English language. The electronic search will be supplemented by manual search of the reference lists of eligible studies and systematic reviews. The searches will be rerun prior to the final analyses and any further studies will be retrieved for inclusion.

\section{Data collection and analysis}

\section{Selection of studies}

The results of the searches in each database will be exported and deduplicated in EndNote X8 version. Two reviewers will independently decide the potential eligibility of each study by title and abstract (keywords where applicable) screening. The full-text articles of the potentially eligible studies will be accessed and further screened against the eligibility criteria by two independent reviewers. Disagreements on the inclusion of studies at both stages will be resolved by consensus with the involvement of a third reviewer, who will also screen a random selection of $10 \%$ of the included papers for 
accuracy. A PRISMA flow diagram will be used to report the screening results.

\section{Data extraction}

A review-specific data extraction form will be devised to extract information of each eligible study in accordance with the review questions. Key information planned to extract include study setting, study population and participant demographics and other baseline characteristics, objectives and details of the programme/intervention (and control if applicable), suggested mechanisms of intervention action, study methodology, participant recruitment, outcomes and measures of outcome assessment, reported/anticipated barriers and facilitators of programme implementation, study limitations and further recommendations. Two independent reviewers will carry out data extraction and any disagreements will be resolved by consensus (with a third author where necessary). Missing data will be requested from study authors.

\section{Quality appraisal}

Since the review aims at integrating evidence from quantitative, qualitative and mixed-methods studies, we will adopt the 'Mixed methods appraisal tool' for the quality appraisal of the eligible studies. ${ }^{36}$ Two independent reviewers will appraise the quality of each study and any disagreements will be resolved by consensus. We will present the results of the quality appraisal for each study and we do not intend to exclude any of the eligible studies based on the quality appraisal in keeping with integrative review methods. ${ }^{34} 37$ Further, since the quality appraisal results will be presented, all eligible studies will be given due prominence irrespective of the data quality.

\section{Strategy for data synthesis}

Given the anticipated methodological pluralism of the potential eligible studies, we will provide a narrative synthesis of the findings from the included studies, structured around answering the main review questions. We will present the summarised information on public health programmes aimed at mental health and wellbeing promotion of young people according to identified thematic areas such as the study setting, target population, type of programme/intervention, programme objectives, reported/proposed mechanisms of intervention action. Further, Whittemore and Knafl's integrative review approach will be used as the guidance for narrative synthesis. ${ }^{34}$

We anticipate that there will be limited scope for metaanalysis owing to the potential wide range of multiple different outcomes measured across a variety of study designs. Thus, a meta-analysis will not be carried out and the programme outcomes will be summarised according to the reported/anticipated change in mental health and well-being (general or disease-specific) of young people, either expressed quantitatively (eg, measures of frequency such as prevalence or incidence, mean scores) or qualitatively. Furthermore, a narrative synthesis of the reported facilitators and barriers in relation to the implementation of public health programmes will be provided.

\section{Patient and public involvement}

Patients or the public are not involved in the design and conception of this systematic integrative review.

\section{Ethics and dissemination}

As only secondary data will be analysed, ethical approval is not required. This is a protocol for a systematic integrative review and the data are not collected yet; hence, there are no data published in a data repository. The results will be disseminated through peer-reviewed publications and/or conference abstracts.

\section{DISCUSSION}

Global evidence suggests that public health programmes, which invest in promotion, prevention and early intervention, not only can reduce the burden of mental ill health, but also make sound economic sense. Thus, in light of the ever-increasing mental health disease burden among young people across the globe, this proposed systematic review focuses on the timely need for identifying the existing gaps in service provision and highlighting the examples of good practice worldwide in relation to public mental health programmes for young people.

The search strategy and the eligibility criteria of this systematic review were carefully designed in collaboration with an information specialist to include a range of related phrases to maximise the sensitivity in our search process. This systematic review was designed following established protocols to maximise rigour and transparency, with involvement of at least two independent reviewers at each stage of the review including data screening, study selection and quality appraisal, which will ensure the robustness of the process.

While considering a number of databases for the literature search without any limits on publication date is a strength of this proposed systematic review, considering publications in English language only is a limitation.

Given that the review findings will be focused on understanding the breadth and depth of global research on public health programmes to promote mental health in young people with a particular emphasis on the facilitators and barriers of programmatic implementation, the insights from this global evidence and good practices are anticipated to make a significant contribution towards improved future development and implementation of public health programmes to promote mental health and well-being of young people across the world.

\section{Author affiliations}

${ }^{1}$ Department of Community Medicine, Faculty of Medicine and Allied Sciences, Rajarata University of Sri Lanka, Saliyapura, Sri Lanka

${ }^{2}$ Cambridge Public Health, University of Cambridge, Cambridge, United Kingdom

${ }^{3}$ Ministry of Health, Nutrition and Indigenous Medicine, Colombo, Sri Lanka

${ }^{4}$ Department of Radiology, Teaching Hospital Kandy, Kandy, Sri Lanka 
${ }^{5}$ Glasgow Caledonian University London, London, United Kingdom

${ }^{6}$ Mental Health Foundation, London, United Kingdom

${ }^{7}$ Faculty of Health and Life Sciences, De Montfort University, Leicester, United Kingdom

Twitter Nuwan Darshana Wickramasinghe @Nu_Wick, Antony Morgan @ antonymorgan3 and Tine Van Bortel @TineVanBortel

Contributors NDW and TVB are joined coprincipal investigators of the study. They conceived the study and have been involved in all aspects of the study. AM, MCW and JF contributed towards the study planning and design. NDW drafted the manuscript and all other authors (TVB, AM, JF, NS, AAK and MCW) edited and revised it critically for important intellectual content. All authors read and approved the final manuscript.

Funding The authors have not declared a specific grant for this research from any funding agency in the public, commercial or not-for-profit sectors.

Competing interests None declared.

Patient and public involvement Patients and/or the public were not involved in the design, or conduct, or reporting, or dissemination plans of this research.

Patient consent for publication Not required.

Provenance and peer review Not commissioned; externally peer reviewed.

Open access This is an open access article distributed in accordance with the Creative Commons Attribution Non Commercial (CC BY-NC 4.0) license, which permits others to distribute, remix, adapt, build upon this work non-commercially, and license their derivative works on different terms, provided the original work is properly cited, appropriate credit is given, any changes made indicated, and the use is non-commercial. See: http://creativecommons.org/licenses/by-nc/4.0/.

ORCID iDs

Nuwan Darshana Wickramasinghe http://orcid.org/0000-0001-6025-6022

Tine Van Bortel http://orcid.org/0000-0003-0467-6393

\section{REFERENCES}

1 Patel V, Flisher AJ, Hetrick S, et al. Mental health of young people: a global public-health challenge. Lancet 2007;369:1302-13.

2 Gore FM, Bloem PJN, Patton GC, et al. Global burden of disease in young people aged 10-24 years: a systematic analysis. Lancet 2011;377:2093-102.

3 World Health Organization. Caring for children and adolescents with mental disorders: setting who directions. Geneva: World Health Organization, 2003.

4 Polanczyk GV, Salum GA, Sugaya LS, et al. Annual research review: a meta-analysis of the worldwide prevalence of mental disorders in children and adolescents. J Child Psychol Psychiatry 2015;56:345-65.

5 Kessler RC, Berglund P, Demler O, et al. Lifetime prevalence and age-of-onset distributions of DSM-IV disorders in the National comorbidity survey replication. Arch Gen Psychiatry 2005;62:593-602.

6 World Health Organization. Child and adolescent mental health. Available: http://www.who.int/mental_health/maternal-child/child adolescent/en/ [Accessed 24 Sep 2018].

7 United Nations Convention on the Rights of the Child. United nations convention on the rights of the child. United Nations, 1989: 1-5.

8 McEwan K, Waddell C, Barker J. Bringing children's mental health "out of the shadows". CMAJ 2007;176:471-2.

9 Department for Education and Department of Health. Transforming children and young people's mental health provision - GOV.UK, 2017 Available: https://assets.publishing.service.gov.uk/government/ uploads/system/uploads/attachment_data/file/664855/Transforming_ children_and_young_people_s_mental_health_provision.pdf

10 Whiteford HA, Ferrari AJ, Degenhardt L, et al. The global burden of mental, neurological and substance use disorders: an analysis from the global burden of disease study 2010. PLoS One 2015;10:e0116820.

11 The National Institute for Healthcare Management Research and Education Foundation. Children's mental health: an overview and key considerations for health system stakeholders. Washington, DC: NIHCM, 2005.
12 Children's Society. The good childhood inquiry: health research evidence. London: Children's Society, 2008.

13 Patel V, Flisher AJ, Nikapota A, et al. Promoting child and adolescent mental health in low and middle income countries. J Child Psychol Psychiatry 2008;49:313-34

14 Kieling $\mathrm{C}$, Baker-Henningham $\mathrm{H}$, Belfer $\mathrm{M}$, et al. Child and adolescent mental health worldwide: evidence for action. Lancet 2011;378:1515-25

15 Jordans MJD, Tol WA, Susanty D, et al. Implementation of a mental health care package for children in areas of armed conflict: a case study from Burundi, Indonesia, Nepal, Sri Lanka, and Sudan. PLoS Med 2013;10:e1001371.

16 Gulliver A, Griffiths KM, Christensen H. Perceived barriers and facilitators to mental health help-seeking in young people: a systematic review. BMC Psychiatry 2010;10:113.

17 McGorry P, Bates T, Birchwood M. Designing youth mental health services for the 21st century: examples from Australia, Ireland and the UK. Br J Psychiatry Suppl 2013;54:s30-5.

18 Gondek D, Edbrooke-Childs J, Velikonja T, et al. Facilitators and barriers to person-centred care in child and young people mental health services: a systematic review. Clin Psychol Psychother 2017;24:870-86.

19 Reardon T, Harvey K, Baranowska M, et al. What do parents perceive are the barriers and facilitators to accessing psychological treatment for mental health problems in children and adolescents? A systematic review of qualitative and quantitative studies. Eur Child Adolesc Psychiatry 2017;26:623-47.

20 World Health Organization. Prevention and promotion in mental health. Geneva: World Health Organization, 2002.

21 Rychetnik L, Frommer M, Hawe P, et al. Criteria for evaluating evidence on public health interventions. J Epidemiol Community Health 2002;56:119-27.

22 Rychetnik L, Hawe P, Waters E, et al. A glossary for evidence based public health. J Epidemiol Community Health 2004;58:538-45.

23 Herman H, Jané-Llopis E. Mental health promotion in public health. Promot Educ 2005;12:42-7.

24 Sturgeon S. Promoting mental health as an essential aspect of health promotion. Health Promot Int 2006;21:36-41.

25 Sawyer MG, Arney FM, Baghurst PA, et al. The mental health of young people in Australia: key findings from the child and adolescent component of the national survey of mental health and well-being. Aust N Z J Psychiatry 2001;35:806-14.

26 Van Bortel T, Wickramasinghe ND, Morgan A, et al. Health assets in a global context: a systematic review of the literature. BMJ Open 2019;9:1.

27 Werner-Seidler A, Perry Y, Calear AL, et al. School-based depression and anxiety prevention programs for young people: a systematic review and meta-analysis. Clin Psychol Rev 2017;51:30-47.

28 Barry MM, Clarke AM, Jenkins R, et al. A systematic review of the effectiveness of mental health promotion interventions for young people in low and middle income countries. BMC Public Health 2013;13:835

29 Das JK, Salam RA, Lassi ZS, et al. Interventions for adolescent mental health: an overview of systematic reviews. $J$ Adolesc Health 2016;59:S49-60.

30 Moher D, Shamseer L, Clarke M, et al. Preferred reporting items for systematic review and meta-analysis protocols (PRISMA-P) 2015 statement. Syst Rev 2015;4:1.

31 Nutbeam D. The challenge to provide evidence in health promotion. Health Promot Int 1999;14:99-101.

32 Dixon-Woods M, Agarwal S, Jones D, et al. Synthesising qualitative and quantitative evidence: a review of possible methods. $J$ Health Serv Res Policy 2005;10:45-53.

33 Jackson N, Waters E, Guidelines for Systematic Reviews in Health Promotion and Public Health Taskforce. Criteria for the systematic review of health promotion and public health interventions. Health Promot Int 2005;20:367-74.

34 Whittemore R, Knafl K. The integrative review: updated methodology. $J$ Adv Nurs 2005;52:546-53.

35 World Health Organization. Child and adolescent health and development. Available: http://www.searo.who.int/entity/child adolescent/topics/adolescent health/en/ [Accessed 24 Sep 2018].

36 Hong QN, Pluye P, Fàbregues S, et al. Mixed method appraisal tool (MMAT) version 2018 user guide. Available: http://mixedmethodsappr aisaltoolpublic.pbworks.com/w/file/fetch/127916259/MMAT 2018 criteria-manual_2018-08-01_ENG.pdf [Accessed 15 Apr 2019].

37 Ganong LH. Integrative reviews of nursing research. Res Nurs Health 1987;10:1-11. 